\title{
EchoGéo
}

25 | 2013

Moyen-Orient : espaces et passeurs du changement

\section{Utopia ou l'anti-Tahrir: le pire des mondes dans le roman de A. K. Towfik}

Delphine Pagès-El Karoui

\section{OpenEdition}

\section{Journals}

Édition électronique

URL : https://journals.openedition.org/echogeo/13512

DOI : 10.4000/echogeo.13512

ISSN : 1963-1197

Éditeur

Pôle de recherche pour l'organisation et la diffusion de l'information géographique (CNRS UMR 8586)

Référence électronique

Delphine Pagès-El Karoui, «Utopia ou l'anti-Tahrir: le pire des mondes dans le roman de A. K. Towfik », EchoGéo [En ligne], 25 | 2013, mis en ligne le 10 octobre 2013, consulté le 10 août 2021. URL : http:// journals.openedition.org/echogeo/13512 ; DOI : https://doi.org/10.4000/echogeo.13512

Ce document a été généré automatiquement le 10 août 2021.

EchoGéo est mis à disposition selon les termes de la licence Creative Commons Attribution - Pas d'Utilisation Commerciale - Pas de Modification 4.0 International (CC BY-NC-ND) 


\section{Utopia ou l'anti-Tahrir: le pire des mondes dans le roman de}

\section{A. K. Towfik}

\section{Delphine Pagès-El Karoui}

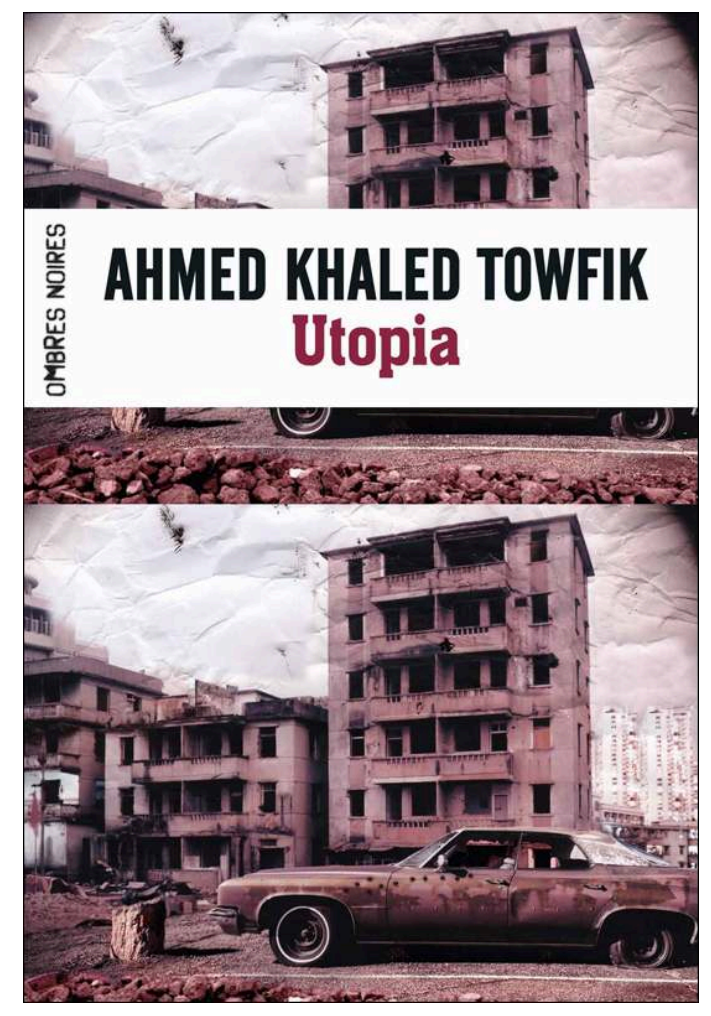

Towfiq, A. K., 2013. Utopia, traduit de l'arabe (Égypte) par Richard Jacquemond, Paris, Ombres noires, $182 \mathrm{p}$.

$\mathrm{Si}$, en Égypte, il y a un espace qui symbolise par excellence le changement socioéconomique impulsé par la mondialisation, c'est bien la cité résidentielle fermée (gated community). Depuis le milieu des années 1990, son essor effréné, dans les périphéries du 
Caire, sur des terrains publics vendus pour une bouchée de pain à des promoteurs privés, a suscité des travaux scientifiques abondants sur la privatisation de la ville et la fragmentation urbaine résultant des logiques néo-libérales (Bouhali, 2008 ; Denis, 2006 ; Florin, 2012). Un écrivain égyptien, Ahmed Khaled Towfik, s'est emparé de cet espace dans un roman d'anticipation, paru avant le mouvement révolutionnaire, en 2008, qui dénonce l'accentuation des inégalités sociales et la disparition des classes moyennes. Utopia $^{1}$ tire son nom de la cité résidentielle fermée sur le littoral méditerranéen, où vivent retranchés les riches égyptiens occidentalisés, tandis que le reste du territoire est réduit à un immense bidonville, où croupissent des populations misérables. L'intrigue, située en 2023, se noue autour de l'incursion dans ces bas-fonds d'un jeune couple d'Utopia qui, pour rompre l'ennui, entend tuer un pauvre et ramener un des ses membres comme trophée.

2 Né en 1962, Ahmed Khaled Towfik qui enseigne toujours la médecine à l'Université de Tantâ, était connu comme un auteur pour littérature adolescente, spécialisé dans l'horreur et la science-fiction. Avec Utopia, devenu un best-seller en Égypte, il a accédé au rang d'écrivain reconnu (Jacquemond, 2013)². Utopia est l'un des romans phares d'un nouvel espace littéraire en Égypte, qui, depuis la sortie de l'Immeuble Yacoubian d'Alaa El Aswany, en 2002, a vu fleurir les best-sellers, plébiscités par un public jeune, éduqué, citadin, et souvent féminin. C'est aussi un symbole de la mondialisation de la littérature, puisqu'il a été publié en anglais, en 2011, par Bloomsbury Qatar Publishing Foundation, une joint-venture britanno-qatarie créée en 2008 qui entend promouvoir la littérature arabe au rang mondial (Jacquemond, 2013).

Face à d'autres auteurs à succès de sa génération, comme Alaa El Aswany ou Khaled $\mathrm{Al}$ Khamissi, auteur de Taxi et de l'Arche de Noé, qui ont eux aussi beaucoup critiqué les travers de leur société, il use d'un procédé littéraire peu courant dans la littérature égyptienne, la dystopie. Contrairement à l'utopie qui, depuis l'ouvrage éponyme de Thomas More, incarne une société imaginaire idéale, celle-ci présente le pire des mondes possibles. Comme il le dit clairement dans son avertissement mis en exergue ${ }^{3}$, Towfiq cherche à alarmer les Égyptiens sur l'état de leur société et l'avenir sombre qui peut en découler. La révolte finale sur laquelle se clôt le roman a été jugée, a posteriori, annonciatrice du processus révolutionnaire. Utopia serait-il un roman visionnaire?

\section{Les deux mondes}

4 La scène inaugurale du livre s'ouvre sur la mise à mort brutale, du haut d'un hélicoptère d'un pauvre hère qui tente de s'introduire illégalement dans Utopia. Ce sont donc bien deux mondes strictement séparés que nous décrit Towfiq. D'un côté, Utopia, ceinte de portails et de barbelés, est protégée par des marines américains à la retraite, qui multiplient les rondes de surveillance et empêchent toute personne sans autorisation d'y pénétrer. À l'intérieur, la cité opulente est divisée entre, d'un côté, le quartier des Jardins qui regroupe les écoles et les lieux de culte (mosquées, églises et synagogues) et de l'autre côté, le quartier des Malls avec ses centres commerciaux gigantesques et ses grandes villas. En contact avec le monde entier, Utopia a même aménagé un aéroport intérieur pour éviter à ses résidents d'avoir à traverser la ville pauvre et ne pas risquer être lynché au passage. La communauté dispose de ses propres journaux, lois et tribunaux, et est gouvernée par un conseil des sages, présidé par les plus grands hommes d'affaires. Le seul clivage qui persiste encore à Utopia semble être 
celui entre les générations : la jeunesse dorée d'Utopia est désœuvrée et dépravée, se livrant ouvertement au sexe et à la drogue à outrance, tandis que leurs parents ont encore l'hypocrisie de cacher leurs vices derrière le voile de la respectabilité et de la religion. Alors que les jeunes ne croient plus en rien, les "vieux " s'accrochent à la religion de peur « de perdre ce qui les distingue, de se retrouver à l'étranger» (p. 22).

5 L'autre monde est celui de l'altérité absolue, puisque ceux qui n'habitent pas dans Utopia sont désignés comme "les Autres». La description de la ville des pauvres est apocalyptique : réduite à un gigantesque bidonville, elle ne dispose plus de services publics. Les réseaux d'électricité et d'égouts ont cessé de fonctionner, le métro a été abandonné et les cinémas ont disparu, le mot même ayant été effacé des mémoires. La population est réduite à la plus grande bestialité, luttant les uns contre les autres pour trouver à manger et échapper aux maladies ou s'évadant de leur condition misérable dans l'alcool, la drogue ou la religion. Une des rares possibilités de travail se trouve à Utopia, où ils occupent les plus basses besognes et sont tolérés pour la journée, mais doivent rentrer le soir, par un bus qui les raccompagne chez eux. Alors que dans la décennie où écrit Towfiq, la frustration sexuelle est intense, liée au retard de l'âge du mariage, faute d'argent, et à l'injonction de l'absence de relations sexuelles hors mariage, en 2023, le sexe est devenu très accessible. Autre changement radical avec l'Égypte de 2008, la disparition des divisions religieuses: la pauvreté partagée a paradoxalement réalisé l'égalité entre les confessions. En revanche, la situation des femmes ne s'est guère améliorée, car elles demeurent toujours à la merci des hommes, réduites au vol ou à la prostitution ${ }^{4}$.

En dépit de leur séparation, les deux mondes se ressemblent et un point commun les relie : la violence, symbole de déshumanisation. Mais de quand date cette profonde transformation de l'Égypte?

\section{La décennie où la classe moyenne a disparu}

7 L'explication est donnée par Gaber, le jeune homme qui va aider les protagonistes à rentrer chez eux, à Utopia. Il évoque la disparition de la classe moyenne lors de la première décennie $\mathrm{du} \mathrm{XXI}^{\mathrm{e}}$ siècle, en raison de très forts taux de chômage et de pauvreté : la classe supérieure «a emporté ce qui restait de la classe moyenne et la société s'est divisée en deux. (...) Mais, il y avait des indicateurs effrayants, tout le monde aurait dû s'en apercevoir» (p.128). Le basculement a eu lieu avec l'effondrement successif des piliers de la rente égyptienne : le tourisme, dont l'activité devenait insuffisante pour faire tourner l'économie; le creusement d'un canal par Israël, court-circuitant le canal de Suez; le tarissement des transferts des émigrés, rentrés du Golfe dont le pétrole était devenu inutile depuis qu'un chimiste américain avait inventé le pyrol en $2010^{5}$. La découverte de ce substitut au pétrole, qui le rendait à peine plus cher que l'eau, a accéléré la chute de l'Égypte. Pour s'approvisionner en pyrol, les élites ont vendu leur passé, soit l'intégralité de leurs monuments pharaoniques aux Américains, en échange d'un contrat de livraison de 50 ans, réservé exclusivement à Utopia et aux autres colonies. Autre conséquence de cette découverte, le renvoi des émigrés du Golfe. "L'économie n'a pas supporté ce fardeau supplémentaire, et l'État s'est lavé les mains de toute responsabilité. Il a abandonné les aides aux pauvres, a tout privatisé. Il n'y avait plus de gouvernement qui s'occupe de nous. Un beau jour, il a cessé de payer les salaires et de faire fonctionner les services. La 
police a disparu. Pendant ce temps, les gens de la classe de vos parents continuaient de s'enrichir. » (p. 99). Ainsi, la disparition de la classe moyenne et de l'État a conduit à la ségrégation des espaces et la régression des hommes vers la violence la plus bestiale. «l'argent du Golfe (avant qu'il ne fonde), le savoir-faire israélien, et la main d'œuvre égyptienne à bon marché » (id.). Pour les gens d'Utopia, Israël n'est plus un ennemi, alors que les pauvres continuent de le percevoir comme tel. Le narrateur affirme avoir de nombreux amis israéliens et il mentionne la présence de synagogues à Utopia. En 2023, la cité fermée est dépendante de l'Occident pour ses importations, non seulement pour le pyrol, mais aussi pour la phlogistine, la drogue qu'elle importe du Danemark et que tous les Egyptiens riches ou pauvres s'arrachent, ainsi que pour le libidafro : sorte de nouveau viagra venu de France. La dépendance aux États-Unis est telle que les nantis d'Utopia se paient, pour assurer leur protection, les services de marines américains à la retraite dont ils sont certains de la loyauté.

Dans le roman, il est souvent question de dissolution de l'identité nationale : "Voilà seize ans que tu n'as d'autre identité que celle de citoyen d'Utopia. La vie facile et l'ennui ont dissous toute appartenance : tu ne reconnais plus l'Américain de l'Égyptien de l'Israélien, vous ne vous différenciez plus les uns des autres » (p. 14). Cet effacement de l'appartenance nationale se traduit par une perte de mémoire. Le jeune narrateur, qui est pourtant cultivé, ne se souvient plus de la guerre de $1973^{6}$ et confond la guerre du Vietnam et avec celle d'Irak. Tout à Utopia dénote les multiples influences de l'occidentalisation capitaliste, et notamment celle du cinéma américain. "On dirait l'affiche de Platoon» (p.11) est la première phrase du livre: le jeune narrateur se fait cette remarque en assistant à la mise à mort d'un intrus tentant de s'introduire dans la cité fermée. Le livre est truffé d'autres références à l'histoire américaine. Pour autant, le narrateur développe un sens aigu de l'appartenance à Utopia, comme un animal à son territoire : "J'ai beau n'appartenir à personne et à nulle part, n'avoir aucun principe, c'est quand même ma patrie » (p.157). Lorsqu'à la fin, la révolte gronde et que le marine leur annonce leur évacuation prochaine, il s'indigne: "Partir? Emigrer? Jamais de la vie. C'est mon territoire, mon univers. Je suis né ici. Mon père a peut-être volé ces droits, mais ils sont miens en vertu de ma naissance et je n'y renoncerai pas pour des Gaber, des mendiants et des filles des rues » (p. 181).

\section{La proie et le chasseur}

Le livre est rédigé à deux voix, structuré en cinq chapitres intitulés successivement Le chasseur et La proie. Le chasseur, dont l'auteur ne livre pas le nom ${ }^{7}$, est un jeune homme de 16 ans, fils d'un grand industriel, égocentrique et décadent qui s'ennuie à mourir, et qui décide de vivre la grande expérience, le meurtre d'un pauvre d'Utopia. Il emmène avec lui son amie Germinal, mais ils seront vite repérés. Au moment, où ils sont identifiés comme habitants d'Utopia et sur le point d'être tués, Gaber les prend sous son aile et parvient à convaincre les autres qu'ils ne sont que des voleurs. Il décide alors de les ramener chez eux. L'odieux narrateur ne connaît aucune rédemption au contact de la misère, puisqu'il viole Safeya, la sœur de Gaber, et tue son frère, une fois qu'il les a ramenés chez lui. Il lui coupe un bras qu'il embaumera. Il pourra alors se vanter avoir accompli son rite d'initiation : "Je suis désormais un homme. Je suis allé là-bas et j'en ai ramené un bras » (p. 171). À l'inverse, l'autre narrateur, Gaber, apparaît 
comme le prophète de la déchéance égyptienne. Il sait que son acte le conduira à la mort, mais il choisit de ne pas tuer les deux d'Utopia, en dépit de sa haine pour eux, car son refus de la violence est la seule chose qui le rattache à l'humanité. Sa mort et le viol de Safeya provoqueront l'étincelle qui allumera les poudres de la révolte ${ }^{8}$.

\section{Un roman visionnaire $?^{9}$}

11 La révolution est évoquée à plusieurs reprises dans le livre, par des références à la révolution iranienne de 1979 et à la révolution française, avec l'image récurrente de la prise de la Bastille. Cependant, l'auteur distille l'impression qu'elle est loin d'avoir lieu, car elle est soit tournée en dérision ${ }^{10}$, soit reléguée à un horizon lointain. Lorsque le jeune homme d'Utopia demande à Gaber pourquoi ils ne se révoltent pas, il lui répond que l'appareil sécuritaire est devenu tellement puissant qu'il dissuade vite les émeutiers.

L'ouvrage de Towfiq qui se clôt sur la révolte des Autres attaquant Utopia, relève davantage de la critique sociale que strictement politique. Ceux qui sont remis en cause ici, ce sont les élites occidentalisées, qui par égoïsme ont vendu leur pays et ont laissé s'appauvrir la majorité de la population. D'ailleurs, quand la révolution est envisagée, c'est aussi dans cette optique sociale: "Les révolutions commencent toujours pas égorger les riches » (p. 99); «Si on fait un jour la révolution, on commencera par manger leurs gros chiens gâtés » (p. 76).

Dans la perspective de la révolution du 25 janvier 2011, des divergences apparaissent avec le scénario imaginé par Towfiq. La pauvreté, le chômage et les inégalités sociales figurent bien parmi les causes principales de l'exaspération populaire, mais la contestation a pris un ton éminemment politique, centré sur la destitution de Moubarak et la chute du régime. De plus, la classe moyenne n'a pas disparu, puisque ce sont ses jeunes qui ont déclenché la protestation. Ces jeunes que les médias occidentaux ont qualifiés d'" occidentalisés" parce qu'ils se servaient d'internet comme outil de contestation, se sentaient avant tout farouchement égyptiens, tout en étant profondément ancrés dans la modernité. Pendant les 18 jours précédant le départ $\mathrm{du}$ raïs, la place Tahrir a éclipsé le contre-modèle d'Utopia: s'emparant d'un espace public symbolique et central, loin des villes nouvelles et leurs cités résidentielles fermées, les Égyptiens ont défié l'autoritarisme et célébré l'unité retrouvée de la nation. Le micro-territoire de Tahrir, organisé comme une petite ville, s'est transformé en laboratoire d'une nouvelle société débarrassée de ses clivages sociaux, politiques, religieux, générationnels, de sexe... (Pagès-El Karoui, 2014). Cependant, le coup d'État de l'armée en juillet 2013, soutenu par une grande partie du peuple, a été suivi par le massacre de nombreux opposants dénonçant la destitution du président islamiste démocratiquement élu. La violence de la répression a ravivé les profonds clivages de la société égyptienne que le moment unanimiste de Tahrir avait voulu masquer (Pagès-El Karoui, 2012). L'ombre menaçante d'Utopia plane sur l'horizon révolutionnaire. 


\section{BIBLIOGRAPHIE}

Bouhali A., 2008. Les compounds cairotes ou la fabrique d'un nouveau mode d'habiter. Des communautés fermées à la ville privatisée ? Mémoire de Master 1, sous la dir. d'O. Pliez, Lyon, ENS-LSH.

Denis E., 2006. Cairo as Neo-Liberal Capital ? From Walled City to Gated communities. In Singerman D., Cairo Cosmopolitan. Poltics, culture and urban space in the new globalized Middle East, Le Caire, The American Univeristy in Cairo Press, p. 47-72.

Florin B., 2012. Les quartiers fermés du Grand Caire. Dimensions urbanistiques et idéologiques d'une forme de ville : nouvelle urbanité ou césure urbaine ? L'Espace Politique [En ligne], 17 | 2012-2, Consulté le 02 septembre 2013. URL : http://espacepolitique.revues.org/2393

Jacquemond R., 2013. The Yacoubian Building and its sisters : reflections on readership and written culture in modern Egypt. In Soliman M., El Hamamsy W., Popular Culture in the Middle East and North Africa: A Postcolonial Outlook, New York, Routledge, p. 223-236.

Pagès-El Karoui D., 2014 (à paraître). 2011, l'odyssée de l'espace public égyptien. In Oualdi M., Pagès-El Karoui D., Verdeil C., Les ondes de choc des révolutions arabes, Beyrouth, IFPO.

Pagès-El Karoui D., 2012. Géographie du changement social en Égypte, EchoGéo [En ligne], 21 | 2012, URL : http://echogeo.revues.org/13204

\section{NOTES}

1. Utopia est le titre arabe du roman. L'usage de mots anglais dans un titre de roman égyptien est rare, mais se développe (cf. le roman policier d'Ahmed Mourad, Vertigo, paru en 2007). Il évoque pour le lecteur arabe l'occidentalisation des élites (Jacquemond, 2013), tant dans leur langage quotidien, truffé de mots anglais, que dans leur mode de vie.

2. Significativement, Utopia a été traduit en français en 2013, non pas chez Actes Sud qui publie la majorité des auteurs égyptiens, mais chez Ombres noires, spécialisée dans les polars venus d'ailleurs, en particulier, des pays du Sud.

3. «L'Utopia évoquée ici est un lieu imaginaire, comme le sont les personnes qui y vivent à l'intérieur et à l'extérieur, même si l'auteur est convaincu qu'elle existera bientôt. Toute ressemblance avec des lieux et des individus de la réalité actuelle est purement fortuite » (Towfiq, 2013, p. 7).

4. Au même moment, des cinéastes dénoncent aussi la violence contre les femmes, qu'il s'agisse de violence conjugale, dans Femmes du Caire de Yousry Nasrallah ou de harcèlement sexuel dans Femmes du bus 678 de Mohamed Diab.

5. Les conséquences du pyrol inventé par Towfiq risquent d'être très proches de celles de l'exploitation du gaz de schiste.

6. Le seul repère temporel du roman est que l'intrigue se situe " cinquante après » la guerre israélo-arabe de 1973, manière pour l'auteur $\mathrm{d}^{1}$ insister sur la centralité de la relation conflictuelle à Israël.

7. Le narrateur s'en justifie : «Qui suis-je? Peu importe mon nom. À quoi bon, quand rien ne distingue les uns des autres?» (p. 15).

8. Les Autres attaquent un convoi de pyrol dans le désert. Ils remplacent discrètement le carburant par des eaux usées qui endommageront les moteurs des avions et voitures d'Utopia. Les habitants sont bloqués, attendant les renforts des hélicoptères américains, quand déferle la masse des révoltés. 
9. Le marketing du livre en Grande-Bretagne s'est fait sur ce thème du roman prémonitoire.

10. «Si la révolution éclate un jour, ce ne sera pas pour la justice sociale, mais pour satisfaire la revendication de tous ceux qui ont été privés de leur droit à la phlogistine » (p. 11).

\section{INDEX}

Keywords : Egypt, revolution, literature, gated community, socio and spatial inequalities.

Mots-clés : Égypte, révolution, littérature, cité résidentielle fermée, inégalités socio-spatiales

\section{AUTEUR}

\section{DELPHINE PAGÈS-EL KAROUI}

Delphine Pagès-El Karoui, d.pageselkaroui@googlemail.com, est Maître de conférences à l'INALCO et membre de l'ANR SYSREMO (Géographies de la mondialisation : émergence d'un système régional au Moyen-Orient, Rennes 2). 